\title{
HELIUM IN THE POLAR WIND
}

\author{
T. I. Gombosi, L. K. Kerr, A. F. Nagy and R. W. Cannata
}

Space Physics Research Laboratory, Department of Atmospheric, Oceanic and Space Sciences, The University of Michigan, Ann Arbor, MI 48109, U.S.A.

\begin{abstract}
The coupled, time-dependent continuity, momentum and energy equations for four charged species $\mathrm{H}^{+}, \mathrm{He}^{+}, \mathrm{O}^{+}$and electrons) moving through a stationary upper neutral atmosphere (composed of $\mathrm{O}_{2}, \mathrm{~N}_{2}, \mathrm{O}, \mathrm{He}, \mathrm{H}$ ) were solved along open geomagnetic field lines using a modified version of the $/ 5 /$ model. The thermospheric densities and temperatures, obtained from the MSIS-86 model $7 /$, were used to calculate neutral-ion sources, sinks, and collision frequencies. The various cross sections and conductivities employed by the $/ 5 /$ model were also updated. The paper presents the first time-dependent solutions for the $\mathrm{He}^{+}$component of the polar wind. Special attention is paid to the solar cycle variation of the ion upflows.
\end{abstract}

\section{INTRODUCTION}

Spacecraft observations of the polar wind and transient upwelling events $/ 10 /$ revealed that $\mathrm{H}^{+}$and $\mathrm{O}^{+}$ions are not the only major constituents of the high speed plasma flows in the topside polar ionosphere: most of the time $\mathrm{He}^{+}$ions also play a very important role, sometimes they even become the major ion species. The early work of Banks and Holzer 12 / showed that light minor ions (lighter than $\mathrm{O}^{+}$) were likely to escape with supersonic velocities in the polar wind. The first attempt to model $\mathrm{He}^{+}$in the polar wind was published in the late $1970 \mathrm{~s} / 12 /$, when steady-state $\mathrm{He}^{+}$density, velocity and temperature profiles were calculated in the presence of a prescribed "classical" $\left(\mathrm{O}^{+}-\mathrm{H}^{+}\right)$polar wind taken from an earlier calculation /11/. Richards et al. /13/ used low speed, interhemispheric, collision dominated, two ion species transport equations to study $\mathrm{He}^{+}$flows in partially depleted flux tubes. They first solved the $\mathrm{O}^{+}$and $\mathrm{H}^{+}$ equations and then used those results to solve the coupled $\mathrm{He}^{+}$and $\mathrm{H}^{+}$equations. They showed that $\mathrm{He}^{+}$ions flow from the winter to the summer ionosphere. Moffett et al. /9/ calculated topside ionospheric $\mathrm{He}^{+}$densities and fluxes using a combination of a low velocity, steady-state momentum equation and the continuity equation. They used this model to study seasonal and solar cycle variations in the $\mathrm{He}^{+}$flux.

Self-consistent steady-state polar wind models with more than one minor ion component run into very serious numerical difficulties. The main problem is that in a steady-state hydrodynamic model each minor ion component has its own sonic point (where the bulk velocity of the given species equals the local sonic speed). In general these sonic points are at different altitudes. It can be shown that in steady-state calculations each sonic point corresponds to a singularity of the differential equation system. In the simplest case, when there is only one minor ion species (and consequently only one singular point), the equations can be solved with some difficulties. This has been done by Banks and Holzer $/ 1,2 /$ and later by Raitt et al. $/ 11 /$. However, the solution of the hydrodynamic transport equation system with two or more singular points is a much more difficult task. On the other hand a time-dependent treatment of the multispecies, transonic hydrodynamic equations does not introduce singularities into the transport equation system.

This paper presents the results of calculations obtained from a significantly modified version of the Gombosi et al. /5/ polar wind model. The new model solves the $\mathrm{He}^{+}$transport equations (continuity, momentum and energy) simultaneously with the $\mathrm{O}^{+}, \mathrm{H}^{+}$and electron conservation equations. This extended model is described in the sections 2 and 3, while the results of the calculations (with special emphasis on the $\mathrm{He}^{+}$flows) are presented and discussed in sections 4 and 5 .

\section{MODEL}

The numerical model simultaneously solves the time-dependent coupled continuity, momentum and energy equations for oxygen, helium and hydrogen ions along diverging open magnetic field lines (the cross-sectional area of a magnetic flux tube, $A$, varies as $A \sim B^{-1}$, where $B$ is the magnetic field strength) and the energy equation for the neutralizing electron gas. It is assumed that the ion and electron gases can be considered to be perfect fluids. The model neglects magnetic field curvature effects and uses a gyration dominated five-moment approximation (assuming that the gyroperiod is the shortest characteristic time scale of the system). It is assumed that the plasma is quasineutral and there is no field aligned current. In this approximation the electron momentum equation can be used to determine the electric field component parallel to the magnetic field.

Burgers' fully linear approximation /13/ was used to calculate the collision terms. The collision frequencies used in the present set of calculations are given in $/ 6 /$. The heat conductivities of the various charged species were taken from $/ 11,12 /$. 
The adopted MSIS-86 $/ 7 /$ neutral atmosphere model includes $\mathrm{N}_{2}, \mathrm{O}_{2}, \mathrm{O}, \mathrm{He}$ and $\mathrm{H}$. Oxygen ions are produced by photoionization and by the $\mathrm{H}^{+}+\mathrm{O} \rightarrow \mathrm{H}+\mathrm{O}^{+}$charge transfer reaction. This latest version of the polar wind model also includes a revised method for calculating photoionization frequencies for atomic oxygen. In the original version 15/, an optically thin F-region was assumed and an altitude-independent photoionization frequency was used. This condition is valid for a wide range of summertime conditions when the sun is high overhead and the level of maximum ionization is well below the lower boundary. However, for high zenith angles, the increased optical path results in a shift upwards in the peak ionization altitude. In the new model this effect has been taken into consideration $16 \%$.

The second process producing oxygen lons is the accidentally resonant $\mathrm{H}_{2}^{+}+\mathrm{O} \rightarrow \mathrm{H}+\mathrm{O}^{+}$charge transfer reaction. $\mathrm{O}^{+}$ is chemically removed by the $\mathrm{O}^{+}+\mathrm{N}_{2} \rightarrow \mathrm{NO}^{+}+\mathrm{N}$ and the $\mathrm{O}^{+}+\mathrm{O}_{2} \rightarrow \mathrm{O}_{2}^{+}+\mathrm{O}$ reactions followed by very fast dissociative recombinations of the $\mathrm{NO}^{+}$and $\mathrm{O}_{2}^{+}$molecular ions. The adopted rate constants were given in $/ 6 \% \mathrm{H}^{+}$ ions are created by the accidentally resonant $\mathrm{O}^{+}+\mathrm{H} \rightarrow \mathrm{O}+\mathrm{H}^{+}$charge exchange process. The $\mathrm{H}^{+}$is removed by the $\mathrm{H}^{+}+\mathrm{O} \rightarrow \mathrm{H}+\mathrm{O}^{+}$charge transfer reaction, which has been discussed above. Helium ions are produced by photoionization; the ionization frequency is $3.87 \times 10^{-8} \mathrm{~s}^{-1}$ for solar minimum and $1.30 \times 10^{-7} \mathrm{~s}^{-1}$ for solar maximum conditions $114 \%$. It should be noted that the atmospheric attenuation of the relevant wavelength band of the ionizing UV radiation is insignificant above $200 \mathrm{~km}$, therefore the present model uses these constant ionization frequencies at all altitudes. $\mathrm{He}^{+}$is removed by reactions with molecular oxygen and nitrogen.

The model flux tube connects two infinite external reservoirs. The ions in the stationary lower reservoir (located at an altitude of $200 \mathrm{~km}$ ) are assumed to be in chemical and thermal equilibrium with the neutral atmosphere, while the electron temperature in this reservoir is set to $1000 \mathrm{~K}$. The upper reservoir (located at an altitude of $8000 \mathrm{~km}$ ) is a stationary, low pressure external medium, in effect representing a topside "vacuum-cleaner." A topside electron heat flux of $5 \times 10^{-3} \mathrm{ergs} \mathrm{cm}^{-2} \mathrm{~s}^{-1}$ is used to simulate energy deposition from the magnetosphere. It is also assumed that there is no ion heat flow between the upper reservoir and the ionospheric flux tube.

The ten, coupled time-dependent partial differential equations were solved with a combined Godunov scheme/CrankNicholson method using dimensional splitting.

\section{RESULTS}

Equilibrium polar wind profiles of hydrogen, helium, oxygen and electron parameters were calculated for solar minimum and maximum conditions. The polar wind flux tube was located at geomagnetic coordinates of $80^{\circ} \mathrm{N}, 00^{\circ}$ W and a solar time of 1200 hours (1700 hours Universal Time). We generated model thermospheres using a daily and averaged $10.7 \mathrm{~cm}$ solar radio flux of 60 (solar minimum) and 180 (solar maximum) and assuming geomagnetically quiet conditions $(\mathrm{Ap}=4$ ) for days 76183 (solar minimum) and 80183 (solar maximum). With these adopted differences the model can account for the major collisional and chemical processes associated with a solar cycle-dependent thermosphere.

Significant differences in the thermospheric gas during solar minimum and maximum exert a major influence on the ion species through chemical and collisional processes. Increases in the molecular species $\left(\mathrm{N}_{2}, \mathrm{O}_{2}\right)$ for solar maximum occur due to greater scale heights associated with higher exospheric temperatures. The higher temperatures (for this case, a $60 \%$ increase) are the direct result of the stronger EUV flux during solar maximum which increases ionospheric heating and ultimately through collisions, the neutral gas. The lighter atomic species react somewhat differently however. In addition to the expected scale height increase for $\mathrm{H}$ and $\mathrm{O}$ at solar maximum, both constituents are subject to transport which further modifies their local concentrations. In the case of the heavier, atomic oxygen, local decreases due to transport are small so the scale height change dominates resulting in an overall local density enhancement at all altitudes at solar maximum. A decrease in $\mathrm{H}$ number densities at solar maximum results from enhanced evaporative escape associated with higher thermal velocities. This different behavior of $\mathrm{H}$ and $\mathrm{O}$ at solar minimum/maximum leads to substantial differences in $\mathrm{H}^{+}, \mathrm{He}^{+}$and $\mathrm{O}^{+}$upwelling behavior. In fact, it is the oppositely-directed responses to solar cycle EUV/UV flux of hydrogen and atomic oxygen which constitute the most effective thermospheric change in altering the high latitude ion outflow character for the summer conditions examined in this study.

The two different thermospheric states corresponding to solar cycle minimum and maximum were used in the model calculations to achieve steady-state model solutions. Figures 1 and 2 summarize steady-state plasma conditions for solar minimum and maximum respectively. In both cases, ions execute upward ambipolar flows due to the polarization electric field which develops between the major ion $\left(\mathrm{O}^{+}\right)$and the more mobile electrons in response to the downward directed pressure gradient. For $\mathrm{O}^{+}$, a very slow, upward-directed flow is present. $\mathrm{H}^{+}$and $\mathrm{He}^{+}$are more easily accelerated upward to supersonic velocities and have flow speeds which are inversely proportional to their respective masses. Density distributions for each ion reflect local production and loss processes with the $\mathrm{O}^{+}$peak located below both $\mathrm{H}^{+}$and $\mathrm{He}^{+}$peaks due to the smaller atomic oxygen scale heights.

In comparing Figures 1 and 2 , a number of different features are apparent in the altitude profiles of the ions which relate directly to changes in thermospheric composition. In the case of the major ion $\mathrm{O}^{+}$, enhanced number densities are apparent at all altitudes for solar maximum. The 'nose' on the solar maximum density profile corresponds to the peak in $\mathrm{O}^{+}$production level which has moved upward (compared to solar minimum) in response to the change in neutral oxygen scale height mentioned earlier. More importantly, enhanced number densities are apparent at all altitudes for solar maximum. This order of magnitude increase in $\mathrm{O}^{+}$results from a combination of two factors. First, higher neutral oxygen scale heights at solar maximum shifts the maximum production level of $\mathrm{O}^{+}$to higher altitudes where loss by reaction with $\mathrm{O}_{2}$ or $\mathrm{N}_{2}$ is less frequent. Secondly, enhanced photoionization rates at solar maximum result in greater $\mathrm{O}^{+}$production. For the season, location and time used in this study, the photoionization frequency at $200 \mathrm{~km}$ increased from $2.0 \times 10^{-7} \mathrm{sec}^{-1}$ to $4.2 \times 10^{-7} \mathrm{sec}^{-1}$ based on the specified increase in F10.7 

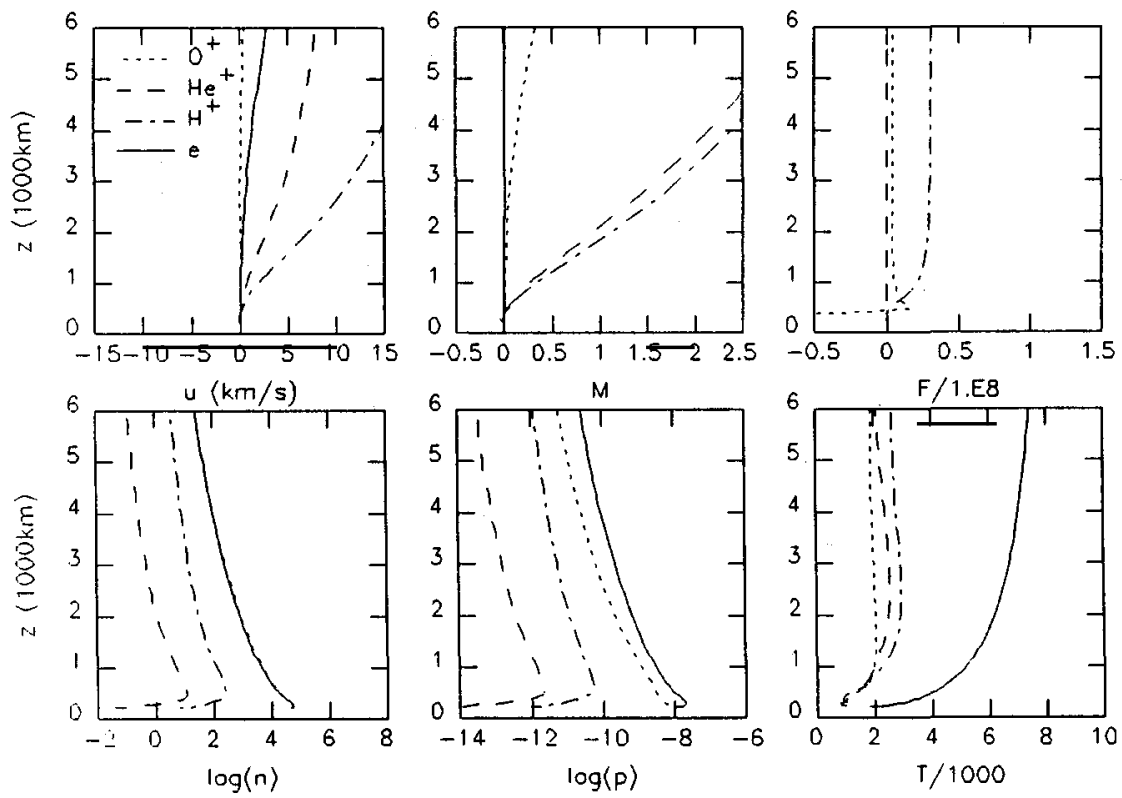

Fig. 1. Calculated velocity, Mach number, flux, particle density, pressure and temperature profiles for solar minimum conditions.
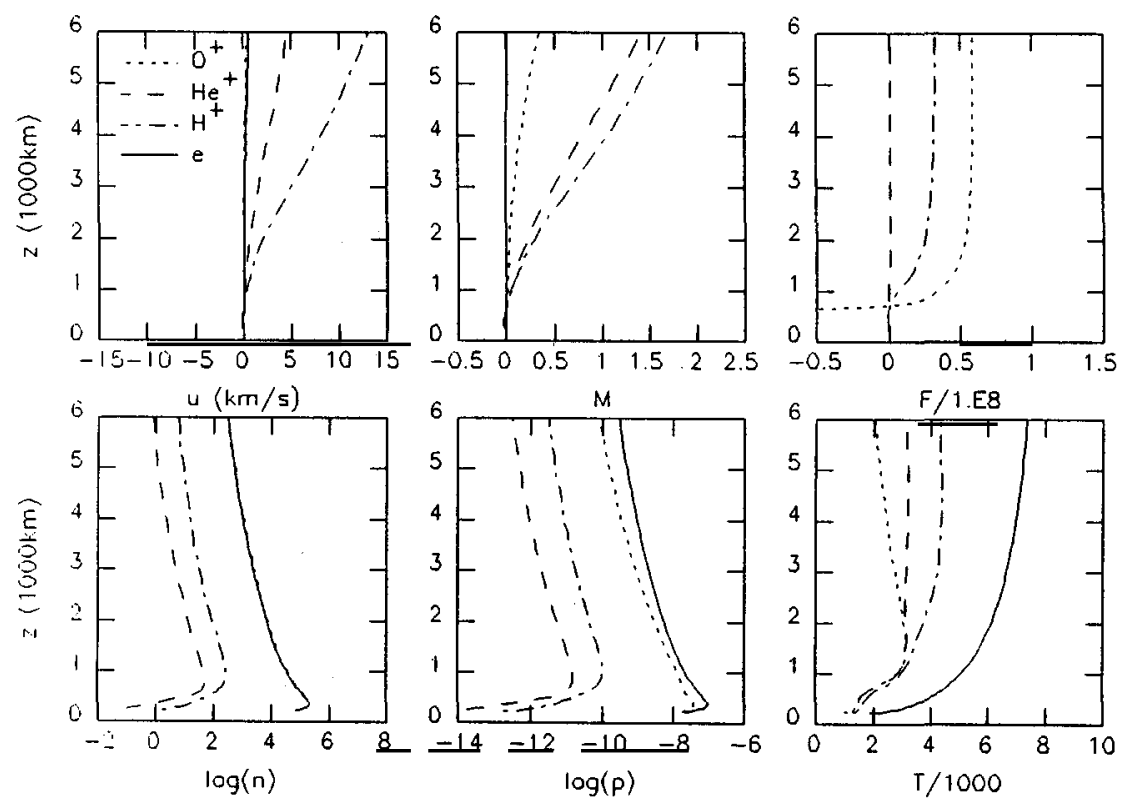

Fig. 2. Calculated velocity, Mach number, flux, particle density, pressure and temperature profiles for solar maximum conditions.

solar radio flux from 60 SFUs to 180 SFUs. Together, the reduced loss and enhanced production of $\mathrm{O}^{+}$during high solar activity substantially increases the $\mathrm{O}^{+}$number density at all altitudes. The $\mathrm{He}^{+}$altitude distributions also vary but to a lesser degree between solar minimum and solar maximum. $\mathrm{He}^{+}$densities are higher at solar maximum due to increased photoproduction rates (about a factor of three greater) due to higher EUV fluxes. For $\mathrm{H}^{+}$, the density and flow velocity profiles shown in Figures 1 and 2 exhibit a more complex behavior. Since generation of $\mathrm{H}^{+}$through the forward charge exchange reaction is most important near the altitude where $n(O)$ equals $n(H)$ (hereafter referred to as the $\mathrm{H} / \mathrm{O}$ crossover altitude), the peak in $\mathrm{H}^{+}$number density shifts upwards as the crossover altitude in the adopted MSIS thermosphere moved from approximately $690 \mathrm{~km}$ (solar minimum) to $1290 \mathrm{~km}$ (solar maximum).

This change in crossover altitude has important consequences for the $\mathrm{H}^{+}$and $\mathrm{He}^{+}$outflow velocities. Once created, hydrogen and helium ions move primarily in response to the ambipolar electric field established by the major ion $\left(\mathrm{O}^{+}\right)$. A lower crossover altitude corresponding to solar minimum, implies a greater vertical distance over which the hydrogen and helium ions can be accelerated by the electric field free from collisions with atomic oxygen. Additionally, since collisions are less frequent above the crossover altitude, both $\mathrm{H}^{+}$and $\mathrm{He}^{+}$experience less drag and 
therefore less momentum loss compared to the solar maximum case. As a result, hydrogen outflow velocities are greater at solar minimum (by a factor of 3 at $2000 \mathrm{~km}$ ) with a somewhat less pronounced variation exibited by the heavier $\mathrm{He}^{+}$. In effect, the upward shift in the collision-dominated regime for the solar maximum thermosphere tends to reduce the effectiveness of both $\mathrm{H}^{+}$and $\mathrm{He}^{+}$acceleration leading to lower outflow velocities at all altitudes for both ions at solar maximum. This behavior modifies the location of sonic flow transition for both species so that supersonic flows are delayed to much greater altitudes during solar maximum. For the cases examined here, transonic altitudes (altitude where flow exceeds Mach 1) differ by almost $4000 \mathrm{~km}$ between solar minimum and maximum. In contrast to the light ion behavior, the field-aligned velocities of $\mathrm{O}^{+}$exhibit almost no solar cycle variation. For this heavier ion, the diffusive flow which develops from the imposed pressure differential along the model flux tube is constrained to approximately constant velocity by the near balance between gravity, friction and the induced polarization electric field.

The distinct variations in density and field-aligned velocities described above for each ion species leads to substantial differences in equilibrium fluxes for both solar cycle cases illustrated in Figures 1 and 2 . All flux profiles have been normalized to the $1000 \mathrm{~km}$ value to account for the radially-divergent flux tube geometry assumed in the model and $\mathrm{He}^{+}$fluxes have been scaled by 10 for comparison with the larger $\mathrm{O}^{+}$and $\mathrm{H}^{+}$fluxes. The $\mathrm{H}^{+}$flux profile shows essentially no dependence on solar cycle conditions reaching an asymptotic value near $5 \times 10^{8} \mathrm{~cm}^{-2} \mathrm{sec}^{-1}$ at $3000 \mathrm{~km}$ for both solar minimum and solar maximum. The near constant $\mathrm{H}^{+}$flux profile can be explained in terms of the opposing responses in $\mathrm{H}^{+}$number density and outflow velocities for solar minimum/maximum periods. Above 4000 $\mathrm{km}$, variations are small and in opposite senses. Slower field-aligned velocities at solar maximum reduce the $\mathrm{H}^{+}$flux between $4000 \mathrm{~km}$ and the $\mathrm{H} / \mathrm{O}$ crossover altitude. Below about $1000 \mathrm{~km}$, both number density and flow velocity are slightly reduced (compared to solar minimum values) and thus act together to reduce $\mathrm{H}^{+}$fluxes during high solar activity. The $\mathrm{O}^{+}$flux also responds to thermospheric changes but in an entirely different manner. In contrast to the hydrogen ion, the $\mathrm{O}^{+}$flux profile shows an order of magnitude variation for solar cycle extremes with fluxes increasing from by slightly one order of magnitude between solar minimum and solar maximum. By comparing the solar cycle number and velocity profiles, this behavior can be directly attributed to the approximate 10-fold increase in $\mathrm{O}^{+}$number density associated with thermospheric compositional changes and solar EUV/UV variations. Additionally, the magnitude of the $\mathrm{O}^{+}$flux at solar maximum exceeds that of $\mathrm{H}^{+}$by a factor of two. For helium ion fluxes, the solar cycle sensitivity falls between the $\mathrm{H}^{+}$and $\mathrm{O}^{+}$flux extremes with only a small increase noted during solar maximum. Although flow velocities are slightly reduced at solar maximum, the reduction is small compared to enhanced $\mathrm{H}^{+}$ densities due to increased photoproduction. As a result, $\mathrm{He}^{+}$fluxes increase slightly at solar maximum. Based on the invariant behavior of the $\mathrm{H}^{+}$flux with solar cycle, a small increase in $\mathrm{He}^{+}$flux for solar maximum, and the large relative increase in $\mathrm{O}^{+}$fluxes at solar maximum, it appears that the polar wind character changes in favor of oxygen ion dominance during solar maximum for the case studied here.

\section{ACKNOWLEDGEMENTS}

This paper was supported by NSF grant ATM-8908183 and NASA grant NAGW-2162. Acknowledgement is also made to the National Center for Atmospheric Research, sponsored by the National Science Foundation, for the computing time used in this research.

\section{REFERENCES}

1. Banks, P.M., and Holzer, T.E., Features of Plasma Transport in the Upper Atmosphere, J. Geophys. Res., 74, $6304,1969 \mathrm{a}$.

2. Banks, P.M., and Holzer, T.E., High-latitude plasma transport: The polar wind, J. Geophys. Res., 74, 6317, $1969 \mathrm{~b}$.

3. Burgers, J.M., Flow equations for composite gases, Academic Press, New York, 1969.

4. Ganguli, S.B., and Palmadesso, P.J., Plasma transport in the auroral return current region, J. Geophys. Res., 92, 8673, 1987.

5. Gombosi, T.I., Cravens, T.E., and Nagy, A.F., A time-dependent theoretical model of the polar wind: Preliminary results, Geophys. Res. Lett., 12, 167, 1985.

6. Gombosi, T.I., Kerr, L.K., Nagy, A.F., and Cannata, R.W., A time-dependent polar wind model with three ion species, J. Geophys. Res., 1990.

7. Hedin, A.E., MSIS-86 thermospheric model, J. Geophys. Res., 92, 4649, 1987.

8. Mitchell, H.G., Jr., and Palmadesso, P.J., A dynamic model for the auroral field line plasma in the presence of field-aligned current, J. Geophys. Res., 88, 2131, 1983.

9. Moffett, R.J., Bailey, G.J., Quegan, S., Rippeth, Y., Samson, A.M., and Seller, R., Modelling the ionospheric and plasmaspheric plasma, Phil.Trans. Roy. London, A, 328, 255, 1989.

10. Moore, T.E., Superthermal ionospheric outflows, Rev. Geophys. Space Phys., 22, 264, 1984.

11. Raitt, W.J., A comparison of the temperature and density structure in high and low speed thermal proton flows, Planet. Space Sci., 23, 1103, 1975.

12. Raitt, W.J., Schunk, R.W., and Banks, P.M., Helium ion outflow from the terrestrial ionosphere, Planet. Space Sci., 26, 255, 1978.

13. Richards, P.G., Schunk, R.W., and Sojka, J.J., Large-scale counterstreaming of $\mathrm{H}^{+}$and $\mathrm{He}^{+}$along plasmaspheric flux tubes, J. Geophys. Res., 88, 7879, 1983.

14. Torr, M.R., and Torr, D.G., Ionization frequencies for solar cycle 21: Revised, J. Geophys. Res., 90, 6675 , 1985 . 